\section{Social and Occupational Functioning Assessment Scale (SOFAS)}

\author{
Bruce Rybarczyk \\ Department of Psychology, Virginia \\ Commonwealth University, Richmond, VA, USA
}

\section{Definition}

The Social and Occupational Functioning Assessment Scale (SOFAS) was derived as a rating scale for Axis V, the clinician's judgment of overall level of functioning, in the Diagnostic and Statistical Manual (DSM) for Mental Disorders 4th Edition (American Psychiatric Association 2000). The SOFAS is a global rating of current functioning ranging from 0 to 100 , with lower scores representing lower functioning. The SOFAS differs from the similar Global Assessment of Functioning (GAF) scale by focusing on social and occupational functioning independent of the overall severity of the individual's psychological symptoms. It also differs from the GAF by including impairments that are caused by both physical and mental disorders, thereby making it a useful assessment tool for traumatic brain injury and other neurological disorders. To be scored, impairments need to be direct effects of mental and physical health problems rather than a consequence of lack of opportunity or environmental limitations. Although more recent DSM systems do not include the multiaxial system with a rating of overall functioning, the SOFAS continues to be utilized as a research instrument in treatment studies.

\section{References and Readings}

American Psychiatric Association. (2000). Diagnostic and statistical manual of mental disorders (4th ed.). Washington, DC: American Psychiatric Association. 